Economía, Sociedad y Territorio, vol. vIII, núm. 27, 2008, 799-808

\title{
El escándalo como alternativa teórica
}

\section{Scandal as a theoretic alternative}

\author{
A ese rizomático, frágil y hermoso camino por donde hoy \\ transita, contingente y autónomo en su decisión, Nexus. \\ Luhmann, Niklas (2007), La SOCIEDAD DE La SOCIEDAD, Herder \\ Editores-Universidad Iberoamericana, MÉXico, 954 PP. \\ ISBN 10: 968-5807-20-5, 13: 978-968-5807-20-3.
}

Problemas acuciantes así como conceptualizaciones radicales y argumentadas están reflejadas y son el resultado de una larga investigación, plasmada, problematizada y no ajena a escándalos que hoy podemos conocer, estudiar y discutir gracias, una vez más, a la magnifica traducción llevada a cabo por Javier Torres Nafarrate del libro de Niklas Luhmann Die Gessellschaft der Gessellschaft, publicado originalmente en 1997 por Suhrkamp Verlag, Frankfurt am Mian, Alemania.

Ciertamente, comentar este libro o intentar una suerte de síntesis de la systemtheorie luhmanniana sería pretencioso y materialmente imposible en una reseña como ésta. Por ello sostengo que, más bien, puede resultar significativo y pedagógico hacer referencia tanto al contexto y discusión sociológica en la cual se desarrolla este programa de investigación, como a algunos conceptos clave de la arquitectura teórica de Luhmann (distinciones, sentido, sistema, entorno, comunicación, persona, observador); con la intención de que provoquen, despierten y orienten el interés del lector no sólo por abordar la lectura de esta obra, sino también por adentrarse en la estimulante y sugestiva propuesta teórica de Niklas Luhmann.

\section{El escándalo como alternativa teórica}

El libro La sociedad de la sociedad constituye, bemoles más bemoles menos, la culminación de un largo recorrido teórico, así como una de las ofertas de reflexión analítica más provocadora y escandalosa de los últimos tiempos. Ello porque la propuesta de la teoría general de los sistemas sociales, elaborada por Niklas Luhmann a lo largo de casi más de treinta años, instala diferencias radicales y se aleja significativamente de la tradición socio- 
lógica, orientándose a la producción teórica de formulaciones conceptuales que crean y generan nuevas condiciones de observación y análisis reflexivo de la realidad social. O, para decirlo en otras palabras, de un planteamiento teórico que se aleja radicalmente de la descripción de descripciones buscando superar la impotencia intelectual de un permanente deber ser que, en última instancia, nos deja siempre más cerca de la moral y de la ideología que de la reflexión científica.

Precisamente si hoy en día se asumen, en línea de principio, las actuales y generalizadas condiciones de incertidumbre y crisis disciplinar -más allá del horizonte temporal limitado por un comienzo y un final- podemos constatar que, en general, para las ciencias sociales y del espíritu los instrumentos epistemológicos y metodológicos con los cuales se acostumbra trabajar, comprender y analizar a la sociedad en el contexto evolutivo denominado modernidad, están agotados y han realizado a cabalidad sus posibilidades, lo cual no significa que los aportes de los clásicos deban negarse.

Pero, ¿qué ocurre cuando la sociología, alejándose de todo afán de cientificidad -incluso de sus clásicos- toma partido por la repetición de viejas fórmulas teóricas para continuar describiendo a la sociedad?, ¿qué pasa cuándo la sociología -contagiada de periodismo y de una moral que apunta a un deber serapuesta por los atributos normativos de la sociedad o continua apostando por el todo y niega las diferencias operativas y funcionales que porta la evolución social?, ¿̇a dónde puede conducir esta lógica cuando -sobre todo actualmente- se constata que quedaron atrás los épicos tiempos del despliegue e institucionalización de la sociología? Cuestiones que, por cierto, afectan no sólo a teorías y métodos sino también, y fundamentalmente, al campo epistémico y a las distintas directrices que articulan la reflexión y observación sociológica de la sociedad.

Si el fenómeno temporal impone una nueva reflexión acerca de nuestros sistemas de percepción, observación, entendimiento y de aquello que designamos realidad, así como de las formas de explicación y de intervención sobre la misma, resulta evidente entonces que el problema de la crisis explicativa de las ciencias sociales en general -y de la sociología en particular- es uno de los principales ámbitos en los que se pone a prueba la epistemología de la modernidad y sus correspondientes discursos éticos, sociales y filosóficos. Entonces resulta inevitable recordar que la función de la sociología -en tanto disciplina científica- es atri- 
buir verdad a los distintos discursos y observaciones que de ella emergen, mas no reificarlos.

Ahora, si en verdad un nuevo fantasma recorre el mundo, probablemente se trate del fantasma de la crisis disciplinar. Lo cual sería también un reflejo de que lo que ha ocurrido es que, al no contar con una teoría de la sociedad -una ciencia de la sociedad como señala Darío Rodríguez- la sociología ha optado por procedimientos y criterios normativos, o por resucitar una y otra vez a los clásicos para reinterpretarlos y dar explicaciones que poco o nada se compadecen con los problemas de contingencia, complejidad y diferenciación que se generan en la sociedad actual.

Precisamente, orientado por estas preocupaciones, Luhmann viene a proponer y se interesa por observar, problematizar y discutir la observación de aquello que sociológicamente podemos ver en la modernidad de la sociedad moderna. Y el punto de partida para una sociología de carácter sistémico -señala el teórico de Bielefeld-se instala precisamente en la clara definición de un concepto de sociedad, cuya carencia nos ha llevado comúnmente a confusiones teórico-semánticas que, en principio, no deberían darse en el ámbito de una disciplina científica.

En tal sentido, en su opera magnum -La sociedad de la sociedad-, Luhmann se plantea como objetivo describir y conceptualizar a la sociedad, con conocimiento pleno de lo que esto implica epistemológica y metodológicamente, lo cual significa sustituir las ideas clásicas sobre la causalidad sistémica, y descartar también la supuesta existencia de estructurales sociales constantes; sobre todo si se reconoce que uno de los puntos críticos de la teoría social ha sido el difuso y poco claro concepto de sistema y sociedad, sobre el cual todavía no ha logrado establecerse un concepto sociológico unívoco.

Lo anterior no implica, en caso alguno, que la propuesta luhmanniana se encuentre aislada o sea ajena a la producción teórica contemporánea; no obstante, es claro que el tipo de soluciones y problematizaciones que ésta avanza, el giro copernicano que nos propone, es sumamente particular y la distingue radicalmente de otros planteamientos teóricos contemporáneos como los de Anthony Giddens, Pierre Bordieu o Jürgen Habermas, toda vez que Luhmann no busca legitimar nuevas descripciones ni establecer puntos de intermediación y consenso entre posiciones ambivalentes, sino más bien, vías de emergencia y escape, con lo cual -como es evidente- trata de construir claves teóricas de lectura, cuya plausibilidad también debe ponerse a discusión. 
Es en este sentido que se puede dimensionar la magnitud teórica de su propuesta, sobre todo si se compara con teorías clásicas como las de Marx, Durkheim, Weber o Parsons, las cuales se caracterizan por redefinir completamente la unidad y el objeto de estudio de la sociología. Mas, lo que distingue la propuesta teórica de Luhmann no radica sustantivamente ni en los fundamentos epistemológicos ni teóricos de su concepción social, sino en la diferencia de énfasis que pone sobre estos factores. Pues mientras el posestructuralismo, los nuevos estudios culturales y la antropología, el deconstuctivismo, la teoría de la información y los estudios tecnológicos en general describen a la sociedad, Luhmann pone el acento en las diferencias, en la autopoíesis y los acoplamientos estructurales de los sistemas que forman la sociedad. Es decir, al teórico alemán le preocupa observar y describir cómo opera y qué hace al sistema, cuáles son las operaciones que los distinguen y constituyen, y también cómo estas operaciones permanecen o cambian, pero sin buscar en lo inmediato soluciones o predicciones apriorísticas orientadas a controlar y/o intervenir las prácticas sociales.

De ahí que Luhmann no le asigne a su propuesta teórica ninguna pretensión de carácter estético, reformista ni mucho menos revolucionario, precisamente porque asume que la crisis teórica es, en la actualidad, de tal magnitud (ideológica) que ya nadie hace intentos por reconstruir la unidad de la disciplina (origen de la pedacería social); sobre todo en un contexto en el que con frecuencia se confunde la investigación teórica con el uso y abuso inflacionario de descripciones y mezclas tanto de textos cuanto de citas, de las teorías clásicas para describir normativamente la realidad (incluso desde fuera de la sociedad), llegando a extremos tales que hasta el pensamiento dialéctico se ha ido transformado poco a poco en lógica del equilibrio.

\section{Decisiones conceptuales}

En este trabajo -el cual como se indicó constituye la exposición sistemática más compresiva de su programa de investigaciónLuhmann señala que se ocupará precisamente del sistema social llamado sociedad moderna, en un intento reflexivo que lógicamente no puede hacerse desde fuera de la sociedad (porque, ¿qué hay fuera de la sociedad?). Teóricamente busca entonces establecer un principio fundamental de un orden distinto con el cual señalar, analítica y fácticamente, el punto de partida de -y parala sociología. Una nueva fundamentación para la teoría y el co- 
nocimiento social, que no persigue ser un punto de llegada (como pretende Habermas con su moral consensual); por el contrario -a diferencia de las propuestas epistemológicas clásicas y de consenso comunicativo-, para Luhmann no hay sujeto u objeto poseedor de razón y juicios a priori. Por ello tampoco establece, como punto de partida, alguna tarea racional del individuo.

Más bien aquello que se puede constatar es lo que operativamente se realiza para observar la sociedad, es decir, observar distinciones. Distinciones que hace el individuo quien -al hacer distinciones- comunica, reflexiona y aprende. Esta paradójica ontología de los fundamentos que desarrolla Luhmann (en tanto análisis de lo dado, es decir, las distinciones) no va encaminada en el sentido justificacionista o trascendental que orienta la reflexión poskantiana (sujeto trascendental cognoscente) y tampoco en el sentido de los principios fundacionales (causa-efecto, fines últimos, espíritu universal) deudores de la filosofía hegeliana; sino más bien en el análisis de las operaciones, de los actos que se observan, se distinguen, se describen y se comunican (toda vez que el acto es ya una distinción). De tal constatación la sociedad deviene, entonces, en el conjunto de todas las comunicaciones posibles, el resto es exterioridad y esta modernidad de orden funcionalmente diferenciado de la sociedad moderna es resultado de la evolución fundamental que se ha desplegado en el tiempo, en las constelaciones del tiempo fundamental, como señala Norbert Elias.

Será sobre la base de la reflexión analítica del observar que Luhmann formaliza el sentido como diferencia entre lo que es y lo que no es (acto/potencia). En esta perspectiva trabaja con el objetivo de establecer un principio básico para los desafíos que enfrenta hoy el análisis sociológico, superando además las clásicas ontologías explicativas de la razón. Por tanto, para esta fenomenología radical -que se sostiene en las distinciones dadasuno de los temas fundamentales del objeto de estudio es el problema de los límites para la sociología, sea desde el punto de vista de la disciplina o desde el problema de los límites de la sociedad. Es decir, ¿̇qué es lo social?, ¿̇cómo se forja/codifica de la realidad social el sentido?, ¿cuál es su origen?

$\mathrm{Al}$ respecto recordemos que para toda la tradición teórica veteroeuropea el fundamento de las cosas tuvo siempre una base racional, cuyo fundamento (ethos) era la razón de fondo de cualquier propuesta discursiva; de ahí, por ejemplo, que para la fenomenología de Husserl la conciencia se constituye en el primer supuesto del conocimiento, mientras que para Heidegger la exis- 
tencia es el ser en sí. Mas para Luhmann el acto, como dato en sí, se fundamenta a sí mismo y no requiere apelar a razones metafísicas o escatológicas. En este sentido se podría sostener que metodológicamente Luhmann no está haciendo teoría, está empíricamente analizando los hechos desde una fenomenología radical que se funda en las distinciones dadas para $-y$ desde allí- generar una posibilidad teórica con sus respectivas claves de lectura.

El resultado de esto es que en su propuesta teórica no hay una búsqueda de etéreos principios de causalidad o finalidad, lo que hay es la observación de las distinciones que comunican y, en tanto son comunicadas, operan sentido pues lógicamente son las operaciones las que conforman el mundo de lo social. Entiéndase bien, el mundo de lo social. A partir de esta radical constatación fenomenológica se construyen las bases epistémicas que guían la arquitectura teórica propuesta por Luhmann, uno de cuyos puntos de arranque es la necesaria formalización del sentido (medio), como diferencia entre lo que es y no es, entre lo que se excluye o incluye, entre aquello que se observa y lo que no puede ser observado (toda vez que empíricamente el ojo que ve, no ve que ve).

Para Luhmann el mundo individual está en el medio sentido. El medio donde transcurre lo social es la distinguibilidad; lo que prevalece allí es sólo lo distinguible y sólo es comunicable lo distinguible. Por tanto el sentido siempre se construye a partir de la realidad que es pura contingencia, hasta que se tematiza y forma parte de lo social (aquello que Parsons signaba como dotaciones sociales de sentido). El sentido aparece, así, situado al interior de la complejidad pero implicado en el desarrollo de la historia -en cuanto mundo de las referencias-, la cual no hubiese sido posible sin el ejercicio de la función de selección atribuida al sentido. El sentido aparece, por tanto, bajo la forma de un excedente de referencias a otras posibilidades de vivencia y acción.

Ahora, el tipo de distinción específico al que se refiere Luhmann es cuando se produce una distinción entre lo que se distingue y lo que queda, pero donde ambas partes siempre están copresentes en la distinción, en la forma como unidad de la diferencia (Spencer Brow ha planteado que toda forma [...] establece un dentro [incluye] y un fuera [excluye]; es decir, establece una diferencia y distingue).

En este entramado sólo la comunicación posibilita una tal $d i$ ferencia y, consecuentemente, la difusión de un grado de distinción. A fin de cuentas -y para efectos operativos- lo único que comunica es la diferencia, pues si no hay diferencia se vuelve a la 
contingencia, a la naturaleza. Se vuelve a la metafísica de lo real, allí donde la realidad es pura contingencia. Ahora, si lo dado (la realidad) es contingencia, para tomar (asir) la realidad hay que hacer diferencias, disminuyendo la complejidad de la contingencia lo cual, sin embargo, creará nuevas complejidades.

Luhmann sostiene que en el mundo de las diferencias encontramos dos operaciones básicas de la distinción: distinciones de la conciencia, que sólo vivencia las distinciones pero no comunican, y distinciones de la comunicación, que al ser comunicadas producen nuevas distinciones y comunicaciones. Aunque ambas operaciones actúan (operan) en el mundo de la diferencia, la conciencia capta la dimensión total pero no la distingue necesariamente, mientras que la comunicación capta diferencias para reproducir nuevas diferencias. Por tanto, operativamente existe una clausura radical de la conciencia, de los sistemas psíquicos (personas), pero evolutivamente surge un orden intermedio que es la comunicación (apertura cognitiva), posibilitando operaciones (sentidos) que crean un orden social (la comunicación crea). De ahí que, empíricamente, es posible incluso sostener que el individuo antes que ser racional es un ser que establece diferencias para luego reflexionar y comunicar.

Al respecto es importante señalar que en el núcleo de esta teoría se distinguen tres tipos de sistemas autorreferentes: los sistemas vivos, los sistemas psíquicos -o relativos a las personas como individuos- y los sistemas sociales. Cada uno de ellos establece una diferencia por la particular forma en que realizan sus operaciones, comunican y reducen la complejidad de sus entornos, de forma tal que los aspectos biológicos son propios de los sistemas vivos, la conciencia es el modo de operación de los sistemas psíquicos y la comunicación es el rasgo característico y definitorio de los sistemas sociales. Precisamente esto permite sostener que la sociedad no es otra cosa que el conjunto de todas las posibles comunicaciones sociales.

\section{Horizonte teórico}

En el desarrollo teórico propuesto por Luhmann para la teoría de los sistemas sociales y para observar la sociedad de la socie$\mathrm{dad}$, conceptos fundamentales como la diferencia que se establece entre sistema/entorno, persona y observador se constituyen también como pilares irreductibles para su arquitectura teórica.

Luhmann sostiene que el entorno es siempre más complejo que el sistema (no olvidemos que todo sistema es una comunica- 
ción estabilizada). Sin embargo, entre sistema/entorno se genera un movimiento dialéctico, pero no en el sentido hegeliano de la determinación causalefecto; sino en el sentido operativo irritación/motivación. Por ello las diferencias y distinciones son siempre operativas y actúan por inclusión/exclusión. De manera tal que todo sistema, al estabilizar un tipo específico de comunicación (código) reduce complejidad, pero al reducir la complejidad del entorno, aumenta la complejidad con el mundo que incluye.

Paradójicamente, para reducir complejidad se debe seleccionar y toda selección es contingente (se decide porque no se puede decidir), de forma tal que para resolver el problema de la contingencia (elección) se establece un tipo de comunicación específica, dando así origen a un sistema (derecho, política, educación, ciencia, arte, etcétera). Lógicamente un sistema es, entonces, una contingencia estabilizada momentáneamente y la sociedad deviene en un nivel de estabilización del sentido, cuyo correlato -semántica histórica- es la civilización e institucionalización de las expectativas que operan como referentes para las personas.

Luhmann señala que las personas no pueden permanecer ni existir sin los sistemas sociales y viceversa. La coevolución condujo hacia ese logro común que es utilizado por los sistemas tanto psíquicos cuanto sociales, ninguno de los cuales puede prescindir de ese logro común y, para ambos, es obligatorio como una forma indispensable e ineludible de complejidad y autorreferencia (logro evolutivo al que llamamos sentido). Así, distintas conciencias (sistemas psíquicos) pueden acoplarse al sistema social a través del medio lenguaje (acoplamiento estructural), puesto que un organismo siempre se acopla a su ambiente dependiendo de la estructura del ambiente y no del ambiente. De ahí que Luhmann no considera aquellos marcos teóricos que subordinan a los individuos a algún tipo de totalidad, ya sea social o trascendente.

En tal sentido, lo primero que se debe establecer para lograr una descripción operacionalmente veraz de la situación que observamos, señala Luhmann, es establecer los límites de la posición del observador y de aquello que se observa, sobre todo si se asume que en la modernidad de la sociedad moderna vivimos en un mundo policéntrico, sin vértices ni cúspide permanente o eterna. Un mundo que no está fuera de nuestro espacio temporal, lo cual ciertamente imposibilita generar certidumbres y establecer prescripciones o normas válidas para toda la humanidad.

En síntesis, por tener su origen tanto en las ciencias naturales y exactas como en una serie de redefiniciones conceptuales de la 
sociología clásica y de la filosofía, la teoría de los sistemas sociales -como método analítico- es aplicable a todas las ciencias sociales, pues además de ofrecer un vocabulario que las unifica, también puede aplicarse en el estudio de los fenómenos sociales, tanto a nivel macro cuanto al micro. Reconocer además que el esfuerzo teórico propuesto por Niklas Luhmann se orienta a integrar diferentes corrientes filosóficas, epistemológicas y sociológicas en pos de una arquitectura teórica que nos permite guiar la observación científica no ya desde una perspectiva ideológica sino -y ante tododesde una clara opción científica y sociológica.

Huelga señalar que el carácter histórico de las ciencias sociales está determinado precisamente por este tipo de conciencia, por las interrogantes que desde allí se generen y por el tipo de observaciones que seamos capaces de formular y operar con respecto a la contingente naturaleza de la realidad social; sobre todo si, a estas alturas, resulta evidente que esa contingencia que nos circunda requiere un nuevo nivel de comprensión -tal y como señala Ilya Prigogine- donde la racionalidad ya no se identifica más con la certeza ni con la probabilidad con la ignorancia, sino que más bien -al final del túnel- siempre hemos de encontrarnos con la complejidad que, en tanto potencia, genera diversas posibilidades aunque nunca certezas.

RAÚl ZAMORANO-FARÍAS Universidad Nacional Autónoma de México Correo-e: rzamorano61@yahoo.it

Recibido: 31 de enero de 2008. Aceptado: 7 de febrero de 2008.

Raúl Zamorano Farías. Es doctor en sociología jurídica por la Universidad de Lecce, Italia. Actualmente se encuentra adscrito al Centro de Estudios Teóricos Interdisciplinarios en Ciencias Sociales de la Universidad Nacional Autónoma de México; profesor invitado de la Università degli Studi di Leche; miembro del Sistema Nacional de Investigadores nivel II (Conacyt-México). Su especialidad es el análisis de la teoría de sistemas. Entre sus publicaciones recientes destacan: "Formas históricas de coordinación social en América Latina: de la teoría de la dependencia al fetichismo neoliberal", Rivista Teoria del Diritto e dello Stato, Torino, Italia, pp. 283-319 (2007); "Modernidad, sociedad y cons- 
titucionalismo en América Latina", Revista Enfoques, 7, Santiago de Chile, pp. 109-145 (2007); "Democracia y constitucionalismo en América Latina. El federalismo mexicano: entre el texto y el contexto", en Entre la utopía y la realidad. Enfoques para una reinterpretación histórica y conceptual de la democracia en América Latina, UAEM-UnAM, Toluca, México, pp. 199-236 (2006); Civilizzazione delle aspettative e democrazia nelle periferie della societá moderna, Roma, Biblioteca Pensa Multimedia, (2004). 